Hydrodynamically Driven Two-Phase Flow,

A Theory of

Hydrodynamically Driven

Dynamic Mix

Judith Binstock 


\section{DISCLAIMER}

Portions of this document may be illegible in electronic image products. Images are produced from the best available original document. 


$$
\text { . }
$$




\section{PREFACE}

This version is a subset of work originally published November 1984, with some deletions/reformatting, but no modifications in the selected original equations. Added notes at the end will comment on some of these equations. 


\title{
Hydrodynamically Driven Two-Phase Flow, A Theory of Hydrodynamically Driven Dynamic Mix
}

by

Judith Binstock

\begin{abstract}
We write the dynamic equations describing the intermixing of two materlals, starting from the conservation laws. The result is a set of equations for mixing driven by the RayleighTaylor buoyancy force, and amplified by terms which incorporate the Richtmyer-Meshkov and Kelvin-Helmholtz instabilities. Making the assumption of pressure equilibration, we arrive at equations which predict that mixing will begin, even in the limit of small initial perturbations, and even in a l-d calculation, when an Interface goes Rayleigh-Taylor unstable, unless the drag or material strength forces are sufficiently large to prevent this. The equations given here may be incorporated into 1-d, 2-d, or 3-d codes, for either Lagrangian or Eulerian formulation. They have the nice feature for Lagrangian codes of keeping the zone mass fixed, thus eliminating the need for rezoning.
\end{abstract}

\section{INTRODUCTION}

How does one describe the interpenetration of two materials? To approach this problem, we go back to first principles and examine the way we describe the motion of one material. The latter is done by assuming a single velocity field and writing the conservation laws for mass, momentum, and energy. Namely, 


$$
\begin{aligned}
& \frac{\partial}{\partial t} \rho=-\nabla_{1} \rho v_{i}, \\
& \frac{\partial}{\partial t} \rho v_{j}=-\nabla_{1} \rho v_{1} v_{j}-\nabla_{1} P_{1 j}, \\
& \frac{\partial}{\partial t} \rho\left(E+\frac{v^{2}}{2}\right)=-\nabla_{1} \rho\left(E+\frac{v^{2}}{2}\right) v_{i}-\nabla_{1} P_{1 j} v_{j},
\end{aligned}
$$

where $E$ is the internal energy per unit mass, and $P_{i j}$ is the pressure tensor.

For a reference frame moving with the velocity field $\vec{v}$, the Lagrangian time derivative is

$$
\frac{d}{d t}=\frac{\partial}{\partial t}+\vec{v} \cdot \vec{\nabla}
$$

Therefore, using (I.2),

$$
\begin{array}{ll}
\frac{d}{d t} \rho+\rho \vec{\nabla} \cdot \vec{v}=0 & ,(\text { from }(I \cdot l a)), \\
\rho \frac{d v}{d t} j+\nabla_{i} P_{i j}=0, & \left(\text { from }(I .1 b)-v_{j} \cdot(I .1 a)\right), \\
\rho \frac{d E}{d t}+P_{i j} \nabla_{1 j}=0,
\end{array}
$$

from $(I .1 c)-v_{j} \cdot\left(I_{.} I b\right)-\left(E-v^{2} / 2\right) \cdot(I .1 a) \cdot$ Because

$$
\vec{\nabla} \cdot \vec{v}=\frac{1}{v} \frac{d V}{d t}
$$

(I.3a) gives

$$
\frac{d m}{d t}=0
$$


for a Lagrangian reference frame. No mass points cross a cell boundary. If we set

$$
P_{i j}=P \delta_{1 j},
$$

we get

$$
\frac{d v}{d t} j=-\frac{1}{\rho} \nabla_{j} P
$$

from $(I .3 b)$,

$$
m \frac{d E}{d t}=-P \frac{d V}{d t},
$$

from (I.3C) and ( $I .6)$, a 11 famillar results.

These equations ( $I .5 a, b, c)$ are used to calculate the hydrodynamic motion with a Lagrangian code.

II. DERIVATION OF EQUATIONS OF MOTION FOR TWO-PHASE FLOW

Now, to describe the interpenetration of two materials, we shall assume two velocity fields, one associated with each material. We shall then write two sets of conservation laws, one for each material.

\section{A. Mass Conservation}

We define new variables as follows. The density of materials 1 and 2 are

$\rho_{1}, \rho_{2}$,

where

$$
\rho_{1}=m_{1} / v, \rho_{2}=m_{2} / v, m=m_{1}+m_{2}, \rho=\rho_{1}+\rho_{2} \text {. }
$$


The associated velocity fields are

$$
\vec{v}+\vec{v}_{1}, \quad \vec{v}+\vec{v}_{2},
$$

where $\vec{v}$ is the velocity of the Lagrangian reference frame.

Then conservation of mass requires

$$
\begin{aligned}
& \frac{\partial}{\partial t} \rho_{1}=-\nabla_{1} \rho_{1}\left(v+v_{1}\right)_{i}, \\
& \frac{\partial}{\partial t} \rho_{2}=-\nabla_{1} \rho_{2}\left(v+v_{2}\right)_{1},
\end{aligned}
$$

or, rearranging terms and using (I.2),

$$
\begin{aligned}
& \frac{d}{d t} \rho_{1}+\nabla_{1} \rho_{1} v_{1_{1}}+\rho_{1} \nabla_{1} v_{1}=0, \\
& \frac{d}{d t} \rho_{2}+\nabla_{1} \rho_{2} v_{2}+\rho_{2} \nabla_{1} v_{1}=0
\end{aligned}
$$

Then by taking (IIA.3.1) + (IIA.3.2) - (I.3a), and using (IIA.1), we get

$$
\vec{\nabla} \cdot\left(\rho_{1} \vec{v}_{1}+\rho_{2} \vec{v}_{2}\right)=0,
$$

which is satisfied if the velocity $\vec{v}$ of the Lagranglan frame is the center-of-mass velocity and

$$
\begin{aligned}
& \rho_{1} \vec{v}_{1}+\rho_{2} \vec{v}_{2}=0 \\
& \text { Now define a drift momentum } \\
& \vec{g} \equiv\left(\rho_{1} \rho_{2} / \rho\right)\left(\vec{v}_{1}-\vec{v}_{2}\right) \\
& =\rho_{1} \vec{v}_{1}=-\rho_{2} \vec{v}_{2},(\text { by }(\operatorname{IIA.4})) .
\end{aligned}
$$


Note that this is the reduced mass times the relative velocity, per unit volume, for the two materlals.

Using (I.4),(IIA.5) in (IIA3.1),(IIA3.2) we get, in the limit of

small volume,

$$
\begin{aligned}
\frac{d m^{2}}{d t}=-\frac{d m}{d t} & =-\int \vec{\nabla} \cdot \vec{g} d V \\
& =-\int \vec{g} \cdot d \vec{A},
\end{aligned}
$$

where the latter integral is over the surface enclosing the volume that contains $m_{1}, m_{2}$. So

$$
\vec{g} \cdot d \vec{A}
$$

gives the rate at which the mass of elther material crosses the area element $\overrightarrow{d A}$. Note that

$$
\frac{d m}{d t}=\frac{d m}{d t} 1+\frac{d m}{d t} 2=0
$$

so that the net mass crossing any area is zero.

\section{B. Momentum Conservation}

Before proceeding to write the full set of conservation laws, we define a new, primed set of variables.

$$
\begin{aligned}
& \mathrm{m}=\mathrm{m}_{1}+\mathrm{m}_{2} \\
& \mathrm{v}=\mathrm{v}_{1}+\mathrm{v}_{2} \\
& \rho_{1}{ }^{\prime}=\mathrm{m}_{1} / \mathrm{v}_{1}, \rho_{2}{ }^{\prime}=\mathrm{m}_{2} / \mathrm{v}_{2} \\
& \mathrm{P}_{1}{ }^{\prime}\left(\rho_{1}{ }^{\prime}, \theta_{1}\right), \mathrm{P}_{2}^{\prime}\left(\rho_{2}{ }^{\prime}, \theta_{2}\right),
\end{aligned}
$$

where $\rho_{1}^{\prime}, \rho_{2}^{\prime}$ are the densities each material would have if it existed as 
small chunks or bubbles, occupying a partial volume. We will write the conservation laws then for two separate regions: the set for material 1 is valid only in the part of the volume occupied by material 1, similarly for material 2. $P_{1}^{\prime}\left(\rho_{1}^{\prime}, \theta_{1}\right)$ represents the pressure in the chunk of material 1 , in its own rest frame. $P_{2}{ }^{\prime}\left(\rho_{2}{ }^{\prime}, \theta_{2}\right)$ represents material 2 , in its own rest frame.

The simplest physical assumption to make relating the two pressures is that of equilibrium, namely,

$$
\theta_{1}=\theta_{2}=\theta
$$

at the same point in space, and the pressures isotropic in each rest frame,

$$
P_{1}^{\prime}\left(\rho_{1}^{\prime}, \theta_{1}\right)=P_{2}^{\prime}\left(\rho_{2}^{\prime}, \theta_{2}\right)=P \text {, }
$$

with the fraction $V_{1} / V$ determined by this pressure equality. Note that there is no real need for either of these assumptions, and some other model could be used to relate $\theta_{1}, \theta_{2}, \rho_{1}^{\prime}, \rho_{2}^{\prime}$.

Writing the mass and momentum conservation laws for material 1 , valid in the partial volume associated with material 1 ,

$$
\begin{aligned}
& \frac{\partial}{\partial t} \rho_{1}^{\prime}=-\nabla_{1} \rho_{1}^{\prime}\left(v+v_{1}\right)_{1} \\
& \frac{\partial}{\partial t} \rho_{1}{ }^{\prime}\left(v+v_{1}\right)_{j}=-\nabla_{1}\left[\rho_{1}^{\prime}\left(v+v_{1}\right)_{1}\left(v+v_{1}\right)_{j}\right]-\nabla_{1} p_{1}^{\prime}{ }_{1 j} \\
& +\mathrm{f}_{12 \mathrm{j}} / \mathrm{v}_{1}
\end{aligned}
$$

where $f_{12} / V_{1}$ is the force per unit volume exerted on material 1 by material 2. From $\left(v_{1} / V\right) \times\left[\left(\right.\right.$ IIB.4b) $-\left(v+v_{1}\right)_{j} \cdot($ IIB.4a $\left.)\right]$, and using(I.2), (IIA.1), (IIA.5), and (IIB.1),

$$
\rho_{1} \frac{d v}{d t} j+\rho_{1} \frac{d v}{d t} l_{j}+g_{i} \nabla_{i}\left(v+v_{1}\right)_{j}+\frac{v_{1}}{v} \nabla_{i} P_{1}{ }^{\prime j j}=f_{12_{j}} / v \text { (IIB.5.1) }
$$


Similarly, by interchanging the labels 1 and 2 , and noting that the drift momentum $g_{1}$ is antisymmetric under this interchange, we write

$$
\rho_{2} \frac{d v}{d t} j+\rho_{2} \frac{d v}{d t}{ }^{j}-g_{1} \nabla_{i}\left(v+v_{2}\right)_{j}+\frac{v_{2}}{V} \nabla_{i} P_{2}^{\prime}{ }_{1 j}=f_{21_{j}} / v \text { (IIB.5.2) }
$$

Note that

$$
\left(f_{12}+f_{21}\right)_{j}=0
$$

because the forces exerted on material 1 by 2 and on 2 by 1 must be equal and opposite.

$$
\begin{aligned}
& \text { Taking (IIB.5.I) +(IIB.5.2) - (I.3b), with (IIB.6), } \\
& \rho_{1} \frac{d v_{1}}{d t}{ }_{j}+\rho_{2} \frac{d v_{2}}{d t} 2_{j}+g_{i} \nabla_{1}\left(v_{1 j}-v_{2 j}\right) \\
& +\frac{V}{V} \nabla_{i} P_{1}^{\prime}{ }_{1 j}+\frac{V_{2}}{V} \nabla_{1} P_{2}^{\prime}{ }_{1 j}=\nabla_{1} P_{1 j} .
\end{aligned}
$$

Defining

$$
\nabla_{1} P^{\prime}{ }_{1 j} \equiv\left(\rho_{1} / \rho_{1}{ }^{\prime}\right) \nabla_{1} P_{1}^{\prime}{ }_{1 j}+\left(1-\rho_{1} / \rho_{1}{ }^{\prime}\right) \nabla_{1} P_{2}^{\prime}{ }_{1 j},
$$

and using (IIA.4), and (IIB.1), (IIB.7) becomes

$$
-v_{1 j} \frac{d}{d t} \rho_{1}-v_{2 j} \frac{d}{d t} \rho_{2}+g_{1} \nabla_{1}\left(v_{1_{j}}-v_{2_{j}}\right)+\nabla_{i} P^{\prime}{ }_{i j}=\nabla_{i} P_{1 j} \cdot \text { (IIB.9) }
$$

Adding (IIB.9) $+\mathrm{v}_{l_{j}} \cdot\left(\right.$ IIA.3.1) $+\mathrm{v}_{2_{j}} \cdot$ (IIA3.2), and using (IIA.5),

$$
\nabla_{i} P_{i j}=\nabla_{i}\left(P^{\prime}{ }_{i j}+g_{i} g_{j} \frac{p}{p 1 \rho 2}\right)
$$


So (I.3b) becomes

$$
\rho \frac{d v}{d t} j+\nabla_{i}\left(P^{\prime}{ }_{1 j}+g_{i} g_{j} \frac{\rho}{\rho_{1} \rho_{2}}\right)=0 .
$$

Note that the extra term $g_{1} g_{j} \rho / \rho_{1} \rho_{2}$ corresponds to a Reynolds stress tensor. Its trace is $\left(\rho_{1} v_{1}{ }^{2}+\rho_{2} v_{2}{ }^{2}\right)$, where $\rho_{1} v_{1}{ }^{2}$ is the contribution to the $\rho v_{i} v_{j}$ tensor from material 1 moving at velocity $v_{1}$ relative to the Lagranglan center-of-mass frame, and similarly for material 2.

To derive the equation of motion for the drift momentum $g_{j}$, write

$$
\begin{aligned}
& \frac{d}{d t} g_{j}=v_{1 j} \frac{d \rho}{d t} l+\rho_{1} \frac{d}{d t} v_{1 j}, \quad \text { (from (IIA.5), } \\
& =-\left(\nabla_{1} g_{i}+\rho_{1} \nabla_{1} v_{1}\right) v_{1 j}-g_{i} \nabla_{i} v_{l_{j}}-g_{i} \nabla_{i} v_{j} \\
& +f_{j} / V-\frac{V}{V}{ }^{1} \nabla_{i} P_{1}^{\prime}{ }_{i j}+\frac{\rho}{\rho} \nabla_{i}\left(P^{\prime}{ }_{i j}+g_{i} g_{j} \frac{\rho}{\rho l \rho 2}\right) \\
& \text { (from (IIA.3.1), (IIB.5.1), (IIA.5), (I.3b)) } \\
& =-\left(\rho_{1} \rho_{2} / \rho\right)\left(\frac{1}{\rho_{1}^{\prime}} \nabla_{1} P_{1}^{\prime}{ }_{i j}-\frac{1}{\rho_{2}^{\prime}} \nabla_{1} P_{2}^{\prime}{ }_{i j}\right)-g_{j} \nabla_{1} v_{1}-g_{1} \nabla_{1} v_{j} \\
& +\left(\rho_{1} / \rho\right) \nabla_{i}\left(g_{i} g_{j} / \rho_{2}\right)-\left(\rho_{2} / \rho\right) \nabla_{i}\left(g_{i} g_{j} / \rho_{1}\right)+f_{j} / V,
\end{aligned}
$$

where we have dropped the subscript 12 on the drag force $f_{j}$.

\section{Energy Conservation}

Now we write the energy conservation equation for material 1 , valid in the partial volume associated with material 1.

$$
\begin{aligned}
\frac{\partial}{\partial t} \rho_{1}{ }^{\prime}\left[E_{1}+\frac{1}{2}\left(\vec{v}+\vec{v}_{1}\right)^{2}\right]= & -\nabla_{1} \rho_{1} \cdot\left[E_{1}+\frac{1}{2}\left(\vec{v}+\vec{v}_{1}\right)^{2}\right]\left(\vec{v}+\vec{v}_{1}\right)_{1} \\
& -\nabla_{1} P_{1}^{\prime}{ }_{1 j}\left(v+v_{1}\right)_{j}+\left(v+v_{1}\right)_{j} f_{j} / v_{l},
\end{aligned}
$$


where $E_{1}$ is the internal energy per unit mass for material 1. Taking $($ IIC.1 $)-\left(v+v_{1}\right)_{j} \cdot($ IIB. $4 b)-\left[E_{I}-\left(\vec{v}+\vec{v}_{1}\right)^{2} / 2\right] \cdot($ IIB. 4a), we get, in the limit of small volume $v$,

$$
m_{1} \frac{d E_{1}}{d t}=-V_{1} P_{1}{ }^{\prime}{ }_{1 j} \nabla_{1}\left(v+v_{1}\right)_{j}-V g_{1} \nabla_{1} E_{1} \text {. }
$$

But

$$
\frac{\mathrm{dm}}{\mathrm{dt}} \mathrm{l}=-\mathrm{v} \vec{\nabla} \cdot \overrightarrow{\mathrm{g}} \quad
$$

from (IIA.6), so that (IIC.2) $+E_{1} \times($ IIC.3) gives

$$
\frac{d}{d t}\left(m_{1} E_{1}\right)=-v_{1} P_{1}{ }^{\prime}{ }_{i j} \nabla_{1}\left(v+v_{1}\right)_{j}-v \nabla_{1} g_{1} E_{1} .
$$

To derive the analogous energy conservation equation for material 2, we interchange the labels 1 and 2 , and utllize the antisymmetry of the drift momentum $g_{1}$, to arrive at

$$
\frac{d}{d t}\left(m_{2} E_{2}\right)=-v_{2^{P}}{ }^{\prime}{ }_{1 j} \nabla_{1}\left(v+v_{2}\right)_{j}+v \nabla_{1} g_{1} E_{2}
$$

Now the total internal energy present in a volume containing mass $m$ is

$$
m E=m_{1} E_{1}+m_{2} E_{2} \text {. }
$$

Therefore, taking (IIC.4.1) +(IIC.4.2) and utilizing (I.5a), (IIB.1), and (IIC.5), we get

$$
\begin{aligned}
m \frac{d E}{d t}= & -v_{1} P_{1}^{\prime}{ }_{1 j} \nabla_{1}\left(v+v_{1}\right)_{j}-v_{2} P_{2}{ }_{1 j} \nabla_{i}\left(v+v_{2}\right)_{j} \\
& +v \nabla_{1} g_{1}\left(E_{2}-E_{1}\right) .
\end{aligned}
$$


III. SUMMARY OF IAGRANGIAN EQUATIONS DESCRIBING THE

INTERPENETRATION OF TWO MATERIALS

We summarize the equations, making the approximation of (IIB.3), namely the pressures equal and isotropic in their rest frames.

$$
\mathrm{m}=\mathrm{m}_{1}+\mathrm{m}_{2}=\text { zone mass } \quad \rho_{1}=\mathrm{m}_{1} / \mathrm{v}, \rho_{2}=\mathrm{m}_{2} / \mathrm{v}
$$

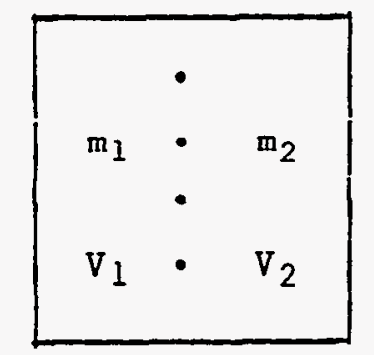

$$
V=v_{1}+v_{2}=\text { zone volume } \rho=m / v
$$

$$
\rho_{1}^{\prime}=m_{1} / v_{1}, \quad \rho_{2}^{\prime}=m_{2} / v_{2}
$$$$
P_{1}{ }^{\prime}\left(\rho_{1}{ }^{\prime}, \theta_{1}\right)=P_{2}{ }^{\prime}\left(\rho_{2}{ }^{\prime}, \theta_{2}\right)=P
$$

$$
E_{1}=E_{1}\left(\rho_{1}^{\prime}, \theta_{1}\right), E_{2}=E_{2}\left(\rho_{2}^{\prime}, \theta_{2}\right)
$$

$$
\frac{d m}{d t}=0
$$

$$
\begin{aligned}
& \rho \frac{d v}{d t} j=-\nabla_{1}\left(P \delta_{i j}+g_{1} g_{j} \rho / \rho_{1} \rho_{2}\right) \\
& m \frac{d E}{d t}=-P \frac{d V}{d t}+V \nabla_{1} g_{1}\left(E_{2}-E_{1}\right)-P V\left(\frac{\rho_{1}}{\rho_{1}}, \nabla_{1} \frac{g_{1}}{\rho_{1}}-\frac{\rho_{2}}{\rho_{2}}, \nabla_{1} \frac{g_{1}}{\rho_{2}}\right)
\end{aligned}
$$

$$
\frac{d m_{1}}{d t}=-\frac{d m^{2}}{d t}=-v \nabla_{1} g_{1}
$$

$$
\frac{d g}{d t} j=-\left(\rho_{1} \rho_{2} / \rho\right)\left(\frac{1}{\rho_{1}}-\frac{1}{\rho_{2}}\right) \nabla_{j} P
$$$$
-g_{j} \nabla_{i} v_{i}-g_{i} \nabla_{i} v_{j}
$$$$
+\frac{\rho}{\rho} 1 \nabla_{1}\left(g_{i} g_{j} / \rho_{2}\right)-\frac{\rho}{\rho} 2 \nabla_{i}\left(g_{i} g_{j} / \rho_{1}\right)
$$$$
+f_{j} / v
$$ 
IV. DISCUSSION OF RESULTS

From (III.2) we see that no rezoning is necessary during the course of a hydrodynamic run. The total mass of any zone is unchanged by the hydrodynamics. The movement of masses of materials 1 and 2 across zone boundaries is described by (III.5), and the amount of material 1 moving across a boundary is compensated by the movement of material 2 in the opposite direction. In (III.4) we see the term $V \nabla_{1} g_{1}\left(E_{2}-E_{1}\right)$, which represents the transport of the internal energy carried by the masses moving across the zone boundaries. The movement of material 1 at velocity $v 1$ relative to the Lagrangian center-of-mass frame, and of material 2 at its relative velocity $v_{2}$, result in (III.3) having a Reynolds stress tensor term whose trace is $\left(\rho_{1} v_{1}{ }^{2}+\rho_{2} v_{2}{ }^{2}\right)$.

The interesting physics is contained in (III.6), which describes the generation and transport of drift momentum $\vec{g}$. The last term, $\vec{f} / v$, is the drag term. Setting it very small, or ignoring it corresponds to having a very large Reynolds number. Setting it infinitely large gives the limiting case of no mix. $\vec{f}$ is always in the direction opposite that of the drift momentum $\vec{g}$, being a frictional force.

Since all but the first of the remaining terms on the right in (III.6) are linear or quadratic in $\vec{g}$, that first term

$$
-\left(\rho_{1} \rho_{2} / \rho\right)\left(\frac{1}{\rho_{1}}-\frac{1}{\rho_{2}}\right) \overrightarrow{\nabla P} \equiv \vec{D}
$$

Is the driving term. Moreover, for an initially unmixed situation, $\frac{\overrightarrow{d g}}{d t}$ remains zero until $\vec{D} \cdot d \vec{A}$ goes positive, if the area vector $\overrightarrow{d A}$ is defined as pointing from material 1 to material 2. This is because a negative value of $\vec{g} \cdot d \vec{A}$ would correspond to material 1 flowing from the region which contains only material 2 to begin with. But this is simply the condition for Rayleigh-Taylor instability, since $\rho 1^{\prime}, \rho 2^{\prime}$ are the actual densities of the respective materials 1 and 2 . Thus the first term on the right in (III.6) is the Rayleigh-Taylor buoyancy force density. Note that (III.6) predicts that mixing will begin at a material interface, even in the limit of small zone size (which corresponds to the limit of small initial perturbation), and even in a l-d calculation, when this driving term goes Rayleigh-Taylor unstable at time $t_{0}$. Regardless of how the 
zoning defining $\left(\rho_{1} \rho_{2} / \rho\right)$ is set up, an expansion of the terms of (III.6) in powers of $\left(t-t_{0}\right)$ gives, for lowest order in $\left(t-t_{0}\right)$,

$$
\frac{d^{2}}{d t}{ }^{2}\left(v_{1}-v_{2}\right)_{j}=\frac{d}{d t}\left[\left(\frac{1}{\rho_{2}}-\frac{1}{\rho_{1}^{\prime}}\right) \nabla_{j} P\right]
$$

at $t=t_{0}$, if the material strength and drag forces are ignored. One way to interpret this driving term is the following. Suppose that any interface has some fuzziness to it. So long as the Rayleigh-Taylor buoyancy force has the stable sign, any relative acceleration of the two materials is in the direction of keeping them separate. Once the Rayleigh-Taylor buoyancy force is in the unstable direction, a relative acceleration of the two materials occurs, causing an intermixing relative velocity to develop. As long as the accelerated material moves through a Rayleigh-Taylor unstable region, it will continue to be accelerated, and so will continue to interpenetrate.

Note also that intermixing as described by these equations (III.1) (III.6) should actually stabilize a 2 -d or 3-d Lagranglan calculation, because any initial perturbation at a Rayleigh-Taylor unstable surface will tend to be smoothed out by the induced mass flow of the two materials across the interface.

The terms linear in dift momentum,

$$
-\vec{g} \vec{\nabla} \cdot \vec{v}-\vec{g} \cdot \vec{\nabla} \vec{v}
$$

in 1-d planar geometry become

$-2 g \frac{\partial v}{\partial x}$

and correspond to the Richtmyer-Meshkov instability. Indeed, in any geometry, the term

$$
-\vec{g} \vec{\nabla} \cdot \vec{v}=-\vec{g} \frac{1}{v} \frac{d V}{d t}
$$


always amplifies the drift momentum when a shock passes through, because the volume strain rate $\frac{1}{\mathrm{~V}} \frac{\mathrm{dV}}{\mathrm{dt}}$ is always negative for shock passage. For the special case of spherical geometry, with

$$
\vec{g}=g(r, \theta, \phi) \theta, \vec{v}=v(r) r, \frac{\partial v}{\partial r}+\frac{3 v}{r}<0,
$$

where the symbol indicates a unit vector, we have

$$
-\vec{g} \vec{\nabla} \cdot \vec{v}-\vec{g} \cdot \vec{\nabla} \vec{v}=-\vec{g}\left(\frac{\partial v}{\partial I}+\frac{3 v}{I}\right)
$$

and the drift momentum $g$, which was just defined perpendicular to the Lagrangian velocity $\vec{v}$, is amplified. This corresponds to a Kelvin-Helmholtz instability. The remaining terms on the right in (III.6), which are quadratic in drift momentum $\vec{g}$, serve to transport the drift momentum associated with the masses of materials 1 and 2 that are crossing the zone boundaries.

V. CONCLUSION

By making the pressure-equilibration assumption of equation (IIB.3), we arrive at equations (III.1) to (III.6), which describe the intermixing of two materfals, driven by the Rayleigh-Taylor buoyancy force. The mixing is amplified by terms which incorporate the features of Richtmyer-Meshkov and Kelvin-Helmholtz instabilities, and damped by drag forces which are represented $\vec{b} y$ the $f / V$ term. These equations predict that mixing will begin, even in the limit of small initial perturbations, and even in a 1-d calculation, when Rayleigh-Taylor instability occurs at an interface, unless the drag or material strength forces are sufficiently large to prevent this.

Note that this derivation describes a mechanism for mixing which can be incorporated into 1-d, 2-d, or 3-d codes, for either Lagrangian or Eulerian formulation. It has the nice feature for Lagrangian codes of keeping the zone mass fixed, thus eliminating the need for rezoning. 
VI. NOTES ADDED SEPTEMBER, 1995

1) The subscripts $i, j$ are vector indices, so that $\nabla_{j} P$ is the $j^{\text {th }}$ component of the gradient of $P$. The Einstein summation convention is assumed, where an index appearing twice implies summation over the values of that index, so that $\nabla_{i} v_{i}$ is $\vec{\nabla} \bullet \vec{v}$, the divergence of the vector $\vec{v}$. $\theta=$ temperature

2) Since equation (IIC.1) is only valid inside material 1 , where $\frac{V_{1}}{V}=1$, the part of the term on the right hand side (RHS) of (IIC.2) with $v_{1}$ can as easily be written $-\mathrm{V}_{P_{1}}^{\prime} \nabla_{i j}\left[\frac{V_{1}}{V} v_{1}\right]_{j}$ instead of the existing term $-v_{1} P_{1}^{\prime}{ }_{i j} \nabla_{i} v_{1 j}$. That the former is the correct generalization for grids too large to resolve the distinct regions of pure materials, we will now show. We will consider the case when $P_{i j}^{\prime}=P \delta_{i j}$. Note that $\mathrm{v}_{1} \bar{\nabla} \bullet \overrightarrow{\mathrm{v}}_{1} \quad$ represents $\iiint_{\mathrm{V}_{1}} \mathrm{dV} \vec{\nabla} \bullet \overrightarrow{\mathrm{v}}_{1}$, the integration of $\bar{\nabla} \bullet \overrightarrow{\mathrm{v}}_{1}$ over the volume $V_{1}$ of distinct regions of material 1 , within the zone of total volume $V$. But physically, this term in (IIC.2) represents the rate at which the volume of material 1 streams out of the Lagrangian zone whose boundary moves at the centerof-mass velocity $\overrightarrow{\mathrm{v}}$, namely $\iint_{\mathrm{A}_{\mathrm{I}}} \frac{\mathrm{V}_{1}}{\mathrm{~V}} \overrightarrow{\mathrm{v}}_{1} \bullet \mathrm{d} \overrightarrow{\mathrm{A}}$, an integral over the zone surface enclosing the full lagrangian volume $V$. Note that $\vec{v}_{1}$, the velocity of material 1 relative to $\vec{v}$, has to be multiplied by $\frac{V_{I}}{V}$, the fraction of the zone volume that is 
material 1. By Gauss's theorem, this integral is $\iiint d V \bar{\nabla} \bullet\left(\frac{V_{1}}{V} \vec{v}_{1}\right)$, and so we generalize $\mathrm{V}$

$\mathrm{V}_{1} \vec{\nabla} \bullet \overrightarrow{\mathrm{v}}_{1} \rightarrow \iiint_{\mathrm{V}_{1}} \mathrm{dV} \bar{\nabla} \bullet \overrightarrow{\mathrm{v}}_{1} \rightarrow \iiint_{\mathrm{V}} \mathrm{dV} \bar{\nabla} \bullet\left(\frac{\mathrm{V}_{1}}{\mathrm{~V}} \overrightarrow{\mathrm{v}}_{1}\right) \rightarrow \mathrm{V} \bar{\nabla} \bullet\left(\frac{\mathrm{V}_{1}}{\mathrm{~V}} \overrightarrow{\mathrm{v}}_{1}\right)$

in order to have equations appropriate to the coarse grid, with cells of volume $V$. Using the notation in this paper, we get for the correct generalization of subgrid multifluid flow, the following new (and hence primed) equations:

$$
\begin{aligned}
& m_{1} \frac{d E_{1}}{d t}=-V_{1} P_{1}^{\prime} \nabla_{i j} v_{j}-V P_{1}^{\prime} \nabla_{i j}\left(\frac{V_{1}}{V} v_{1}\right)_{j}-V g_{i} \nabla_{i} E_{1} \quad \text { (IIC.2)' } \\
& \frac{d}{d t}\left(m_{1} E_{1}\right)=-v_{1} P_{1}^{\prime}{ }_{i j} \nabla_{i} v_{j}-v P_{I}^{\prime}{ }_{i j} \nabla_{i}\left(\frac{v_{1}}{V} v_{1}\right)_{j}-v \nabla_{i} g_{i} E_{1} \quad \text { (IIC.4.1). } \\
& \frac{d}{d t}\left(m_{2} E_{2}\right)=-v_{2} P_{2}^{\prime}{ }_{i j} \nabla_{i} v_{j}-V P_{2}^{\prime}{ }_{i j} \nabla_{i}\left(\frac{V_{2}}{V} v_{2}\right)_{j}+V \nabla_{i} g_{i} E_{2} \quad \text { (IIC.4.2)' } \\
& m \frac{d E}{d t}=-v_{1} P_{1}^{\prime} \nabla_{i j} v_{j}-V P_{1}^{\prime} \nabla_{i j}\left(\frac{V_{1}}{V} v_{1}\right)_{j} \\
& -v_{2} P_{2}^{\prime} \nabla_{i j} v_{j}-V P_{2}^{\prime} \nabla_{i j}\left(\frac{V_{2}}{V} v_{2}\right)_{j} \\
& +V \nabla_{i} g_{i}\left(E_{2}-E_{1}\right) \\
& m \frac{d E}{d t}=-P \frac{d V}{d t}+V \nabla_{i} g_{i}\left(E_{2}-E_{1}\right)-P V \nabla_{i} g_{i}\left(\frac{1}{\rho_{1}}-\frac{1}{\rho_{2}^{\prime}}\right)
\end{aligned}
$$

Note that for clarity we have numbered these equations in a nonstandard fashion, so that the reader can easily determine which equations they supercede, namely the corresponding unprimed equation numbers.

We now show that the last term on the RHS of (III.4)' exactly cancels out the contribution of transport effects to $-\mathrm{P} \frac{\mathrm{dV}}{\mathrm{dt}}$, so that only real compression, and actual transport of internal energy the second term on the RHS, namely 
$V \nabla_{i} g_{i}\left(E_{2}-E_{1}\right)$ ] contribute to the change in internal energy, as the physics requires. Since

$V \nabla_{i} g_{i}\left(\frac{1}{\rho_{1}^{\prime}}-\frac{1}{\rho_{2}^{\prime}}\right) \rightarrow \iiint_{V} d V \vec{\nabla} \cdot \vec{g}\left(\frac{1}{\rho_{1}}-\frac{1}{\rho_{2}}\right)$,

which by Gauss's theorem

$=\iint_{A_{L}} \vec{g}\left(\frac{1}{\rho_{1}}-\frac{1}{\rho_{2}}\right) \cdot d \vec{A}$.

which, by definition of the drift momentum $\vec{g}$,

$=\iint\left(\frac{V_{1}}{V} \vec{v}_{1}+\frac{V_{2}}{V} \vec{v}_{2}\right) \cdot d \vec{A}$

$\mathrm{A}_{\mathrm{L}}$

$=\left[\begin{array}{l}\text { The rate at which the volume } \mathrm{V}=\mathrm{v}_{1}+\mathrm{v}_{2} \\ \text { leaves the Lagrangian (constant mass) } \\ \text { zone }\end{array}\right]$.

it follows that $V \nabla_{i} g_{i}\left(\frac{1}{\rho_{1}}-\frac{1}{\rho_{2}}\right)$ is exactly equal, and opposite in sign, to the contribution to the rate of change of the Lagrangian zone volume, $\frac{d V}{d t}$, due to the exchange of equal masses (of dissimilar microscopic densities) crossing the cell boundaries and causing the Lagrangian interface to move. As a special case, when neither constituent material is compressible, equation (III.4)' limits correctly to $m \frac{d E}{d t}=V \nabla_{i} g_{i}\left(E_{2}-E_{1}\right)$.

Note how the drag force $\overrightarrow{\hat{f}}$ has dropped out of equation (IIC.2), and all subsequent equations involving the time rate of change of internal energy $E_{1}, E_{2}$, or $E$. This is because of the tacit assumption that there is no turbulence, even on the subgrid scale, for this simple model. 